\title{
1 Evaluation of the causal effects of blood lipid levels on gout with 2 summary level GWAS data: two-sample Mendelian 3 randomization and mediation analysis
}

5 Xinghao Yu ${ }^{\text {a }}$, Haimiao Chen ${ }^{\mathrm{a}}$, Shuiping Huang ${ }^{\text {a, b, * }}$, Ping Zeng a, b *

$7 \quad{ }^{a}$ Department of Epidemiology and Biostatistics, School of Public Health, Xuzhou Medical

8 University, Xuzhou, Jiangsu 221004, China

$9 b$ Center for Medical Statistics and Data Analysis, School of Public Health, Xuzhou Medical

10 University, Xuzhou, Jiangsu 221004, China

13 Correspondence to: Ping Zeng, Department of Epidemiology and Biostatistics, School of 14 Public Health, Xuzhou Medical University, Xuzhou, Jiangsu, 221004, China; Center for 15 Medical Statistics and Data Analysis, School of Public Health, Xuzhou Medical University, 16 Xuzhou, Jiangsu, 221004, China 


\section{Abstract}

20 Objective: Many observational studies have identified that gout patients are often comorbid with dyslipidemia, which is typically characterized by a decrease in high-density lipoprotein cholesterol (HDL) and an increase in triglycerides (TG). However, the relationship between 23 dyslipidemia and gout is still unclear.

Methods: We first performed a two-sample Mendelian randomization (MR) to evaluate the causal effect of four lipid traits on gout and serum urate based on summary association statistics available from large scale genome-wide association studies (up to 100,000 for lipid, 69,374 for gout and 110,347 for serum urate). We adopted multivariable Mendelian randomization to estimate the causal effect independently. We also assessed the mediated effect by serum urate between lipids and gout with a mediation analysis. The MR results were validated with extensive sensitive analyses.

Results: Genetically lower HDL was positively associated with the risk of gout and serum urate concentration. Each standard deviation (SD) $(\sim 12.26 \mathrm{mg} / \mathrm{dL})$ increase was genetically associated with an odds ratio of gout of 0.75 (95\% CI $0.62 \sim 0.91, p=3.31 \mathrm{E}-3)$ and with a $0.09 \mathrm{mg} / \mathrm{dL}(95 \% \mathrm{CI}:-0.12 \sim-0.05, p=7.00 \mathrm{E}-04)$ decrease in serum urate concentration. Genetically higher TG was positively associated with the serum urate concentration. Each SD $(\sim 112.33 \mathrm{mg} / \mathrm{dL})$ increase was genetically associated with a $0.10 \mathrm{mg} / \mathrm{dL}(95 \% \mathrm{CI}: 0.06 \sim 0.14$, $p=9.87 \mathrm{E}-05)$ increase in serum urate concentration. Those results were robust against various sensitive analyses. In addition, the multivariable Mendelian randomization confirmed the independent effect of HDL and TG on the gout/serum urate after adjustment for the other lipids. Finally, the mediation analysis showed that both HDL and TG could indirectly affect gout morbidity via the pathway of serum urate. The mediation effect accounted for about $13.0 \%$ or $28.0 \%$ of the total effect of HDL and TG, respectively.

Conclusion: Our study confirmed the causal associations between HDL/TG and gout/ serum urate. Furthermore, the effect of HDL or TG on gout could also be mediated by serum urate.

Keywords: Dyslipidemia; Gout; Serum urate concentration; Mediation analysis; Mendelian randomization; Instrumental variable 


\section{$48 \quad$ Key Messages}

49 - Epidemiological studies have identified an accompanying association between lipid and 50 gout. However, whether the association is causal is unclear.

51 - Mendelian randomization with genetic variants as instrumental variables is a useful tool 52 facilitate the validation of a causal relationship for modifiable risk factors.

- The direct and indirect effects of lipids on gout, controlling for the serum urate concentration, can be estimated by a mediation analysis with serum urate serving as a mediator.

- We confirmed that elevated HDL levels can directly and indirectly lead to the decreased

57 risk of gout, whereas elevation of TG levels can directly and indirectly elevate the risk of 58 gout. 


\section{Introduction}

61 Gout is a common and complex form of arthritis with anyone at risk. It is characterized by sudden, severe attacks of pain, swelling, redness and tenderness in the joints, usually resulting from the chronic elevation of uric acid levels and the deposition of urate crystals in the body (e.g. joints and kidneys). Gout is manifested as rheumatism, gouty arthritis or gouty nephropathy, and is predominantly related to hyperuricemia due to metabolic disorders or reduced excretion of uric acid. Gout is typically an acute onset of single-joint synovitis in peripheral joints, causing extreme pain, whereas it is self-limiting and usually resolves within a few days (1-3). The global burden of gout is substantial, with the prevalence of gout ranging from $0.1 \%$ to $10 \%$ and the annual incidence from 30 to 600 cases per 100,000 individuals. In addition, gout is associated with several complex diseases (e.g. metabolic syndrome (4), cardiovasculopathy (5) and nephropathy (6)). Recently, gout per se was identified to be an independent risk factor in cardiovascular diseases $(7,8)$.

Beyond sustained hyperuricemia (9-11), many factors as genetic, environmental and social, e.g. diet, medication, comorbidities and exposure to heavy metals as cadmium and lead (12), are also involved in the pathogenesis of gout(13). In addition, some empirical studies have indicated a positive correlation between dyslipidemia and the risk of gout $(14,15)$, as evidenced in the increased prevalence and incidence of gout in people with dyslipidemia (16). Dyslipidemia is characteristic of aberrant blood lipids, including abnormalities of high-density lipoprotein cholesterol (HDL), low-density lipoprotein cholesterol (LDL), total cholesterol (TC) and triglycerides (TG). The third National Health and Nutrition Examination Survey of the United States reported that in gout patients the risk of hypertriglyceridemia was doubled and the odds ratio (OR) of low HDL was 1.60 after adjustment for age and gender (4), implying elevated HDL may lead to the decreased risk of gout. A cross-sectional study in 4,053 young adults revealed that the elevation of serum urate was associated with metabolic abnormalities (e.g. hyperinsulinemia, hypertension, dyslipidemia, and obesity) (17). In a study of the Japanese population, glucokinase regulatory protein was associated with decreased

87 fasting blood glucose levels, elevated TG and serum urate concentrations, and was associated with dyslipidemia (18). On the contrary, some studies indicated that there was no significant difference in blood lipid levels between gout patients and normal controls (19). 
90 These controversies over the relationship between dyslipidemia and gout may be partly 91 attributable to uncontrolled/unmeasured confounders in observational studies. Additionally, 92 doubt remains as to whether dyslipidemia is subsequent or consequent to gout. In case of 93 limited observational evidence, Mendelian randomization with genetic variants as 94 instrumental variables can facilitate the validation of a causal relationship for modifiable risk 95 factors (20-22). In brief, Mendelian randomization holds that genetic variants associated with 96 an exposure would also be associated with an outcome through the exposure route if the 97 exposure is causally associated with the outcome.

Mendelian randomization employs genetic variants as instruments, and is often less

99 susceptible to confounders compared with conventional observational studies in that genetic 100 variants are randomly allocated at conception (23). This randomization process is analogous 101 to the profile in a randomized controlled trial wherein participants are subjected to 102 randomized classification and varied exposure. The substantial variation of outcome between 103 groups provides evidence of the putative causal effect of the exposure on the outcome. In this 104 sense, Mendelian randomization is at the interface of experimental and observational studies 105 and is also referred to as the natural randomized trial $(24,25)$ to generate evidence in support 106 of the potential causal effect of an exposure.

107 Indeed, Mendelian randomization has become a potent and effective statistical method for 108 causal inference in observational studies, owing to recent achievements of genome-wide 109 association studies (GWASs) (26-34). In the present study, we employed single nucleotide 110 polymorphisms (SNPs) associated with lipid traits as instrumental variables to determine their 111 causal associations with gout. We conducted the largest and most comprehensive two-sample 112 Mendelian randomization analysis to date with summary statistics from large-scale GWASs 113 with $\sim 180,000$ individuals for lipid traits (i.e. HDL, LDL, TC and TG) (35), 2,100 cases for 114 gout (36) and $\sim 110,000$ individuals for serum urate (36). 


\section{Materials and Methods}

\section{GWAS data}

117 The genetic data sets of the four lipid traits (i.e. HDL, LDL, TC and TG) were available from 118 the Global Lipids Genetics consortium (GLGC) (35) (http://csg.sph.umich.edu/). After 119 stringent quality control of genetic variants and individuals, 2,250,000 SNPs remained 120 including genotyped and imputed from 188,577 individuals of European ancestry. Each of the 121 four lipids was adjusted for available covariates (e.g. age, age ${ }^{2}$ and gender) and then the 122 resultant residuals were quantile normalized to be a standard normal distribution. The 123 association was finally performed for each SNP with a linear additive regression model. For 124 each lipid trait we yielded its summary association statistics (e.g. effect allele, effect allele 125 frequency, marginal effect size, standard error, $\mathrm{p}$ value and sample size).

126 We acquired genetic data sets for gout and serum urate concentration from the Global Urate 127 Genetics Consortium (http://metabolomics.helmholtz-muenchen.de/) (36). After stringent 128 quality control, a total of 69,374 (2,115 cases and 67,259 controls) European individuals and 2,538,056 genotyped and imputed SNPs remained for gout, and 110,347 individuals and

$1302,450,547$ genotyped and imputed SNPs were reserved for serum urate. The association 131 between each SNP and gout/serum urate was analyzed with an additive logistic/linear 132 regression while adjusting for other available covariates (e.g. age and gender). Again, the 133 summary association statistics of gout/serum urate (e.g. effect allele, marginal effect size,

134 standard error, $\mathrm{p}$ value and sample size) were downloaded and refined. The GWAS genetic 135 data sets used in our study are summarized in Table 1.

\section{Selection of instrumental variables}

137 Based on our prior studies $(28,37)$, we selected instrumental variables for lipids with the use 138 of the clumping procedure of PLINK (version v1.90 b3.38) (38). When clumping, we set both 139 the primary significance level and the secondary significance level for index SNPs to 5.00E-8, 140 the linkage disequilibrium and the physical distance to 0.01 and $1,000 \mathrm{~kb}$, respectively. We 141 then selected the lipid-specific index SNPs as instrument variables and finally generated 103 142 SNPs for HDL, 50 SNPs for LDL, 60 SNPs for TC and 60 SNPs for TG. As per previous MR 143 literature $(28,31,39-48)$, we further examined pleiotropic association by removal of SNPs 144 which may be potentially associated with gout or serum urate. Specifically, we excluded index 
145 SNPs which had an adjusted p value of less than 0.05 after Bonferroni correction or residing 146 within the gout- or serum-urate- associated loci (i.e. $1 \mathrm{Mb}$ in the physical distance). Of note,

147 the removal of SNPs with pleiotropic effects is an authenticated manner to ensure the validity

148 of Mendelian randomization (37).

\section{Estimation of causal effect and sensitivity analyses}

150 We subsequently employed Mendelian randomization to determine the causal relationship 151 between lipids and gout/serum urate. First, we calculated the proportion of phenotypic 152 variance explained (PVE) by instruments and computed the $F$ statistic for the four blood 153 lipids to quantitatively verify whether the selected index SNPs were strong instruments (28, $15437,49,50)$. For the four lipid traits, we initially conducted the inverse variance weighted 155 (IVW) method to estimate their causal effects on gout/serum urate (51). We then conducted 156 several sensitivity analyses to assess the validity of our IVW Mendelian randomization results: 157 (1) weighted median-based method (52) and maximum likelihood method (53); (2) 158 leave-one-out (LOO) cross-validation analysis $(37,49)$ to validate whether there were 159 instrumental outliers that can substantially impact the causal effect estimates; (3) MR-Egger 160 regression to further evaluate horizontal pleiotropic effects of instrumental variables (54); (4) 161 reverse causal analysis to assess whether gout/serum urate exhibited a causal effect on lipids 162 using instrumental variables of gout or serum urate, since the determination of the causal 163 direction is very important in Mendelian randomization (55); (5) multivariable Mendelian 164 randomization analysis $(28,37,56)$ to investigate the relationship between one lipid trait (e.g. $165 \mathrm{HDL}$ ) and gout/serum urate while adjusting for the effects of other lipids (e.g. LDL, TC and 166 TG).

167 Mediation analysis to explore the mediation effect of serum urate in the path from lipids to gout

169 Emerging evidence demonstrated that elevated serum urate is the most important single-risk 170 factor and urate-lowering therapy is frequently recommended in the clinical management of 171 gout patients (57). Nonetheless, on the grounds of the causal relationships between lipids and 172 serum urate identified in our analysis, a natural and immediate problem arose as to whether 173 serum urate can mediate the effect of lipids on gout (Fig. 1 C), or rather, do lipids have an 174 indirect effect on gout via serum urate? To address this problem, we further performed a 
175 mediation analysis with serum urate serving as a mediator and estimated the mediating effect 176 of serum urate (i.e. the indirect effect of lipids) (58-62). The estimation and hypothesis testing

177 of the mediation effect were also implemented within the framework of Mendelian 178 randomization based on summary association statistics of lipids, serum urate and gout (Table 1791 and Fig. 1 D). Briefly, we estimated the causal effect of lipids on serum urate with IVW 180 methods, and estimated the causal effect of serum urate on gout by the multivariable 181 Mendelian randomization analysis. Such an analysis is also referred to as network Mendelian 182 randomization $(63,64)$. The mediation effect was evaluated with the product method and was 183 tested with the Sobel test (65) or the Bootstrap test (66). The details of the mediation analysis 184 were demonstrated in Additional file 1.

185 Our statistical analysis was mainly conducted within the R (version 3.5.2) software. As there 186 were four exposures and two outcomes in our Mendelian randomization analysis, the 187 statistical significance level was adjusted to 6.30E-03 $(=0.05 / 8)$ to incorporate the issue of 188 multiple hypothesis testing. In addition, since participants had provided written informed 189 consent for data sharing as described in each of the original GWASs; ethical review was 190 omitted in our study. 


\section{Result}

\section{Causal effect of lipids on gout}

193 We screened out 92, 48, 58 and 53 lipid-specific instrumental variables for HDL, LDL, TC 194 and TG when evaluating their associations with gout. Together, these instrumental variables 195 accounted for a total of 5.7\%, 3.1\%, $2.6 \%$ and $4.3 \%$ phenotypic variances for HDL, LDL, TC 196 and TG, respectively. The $F$ statistics for all these SNPs were greater than 10 (Additional file 197 2: Tables S1-S4), suggesting that weak instrument bias was potentially null in our analysis. 198 The Cochran's Q test $(67,68)$ showed that there was little evidence of instrumental 199 heterogeneity for HDL $(p=0.945)$, TC $(p=0.179)$ or TG $(p=0.379)$. However, an

200 instrumental heterogeneity was observed for LDL at the marginal significance level of 0.05 ( $p$ $201=0.047)$. Therefore, we employed the fixed-effects IVW method for HDL, TC and TG, and 202 applied the random-effect IVW method for LDL when estimating the causal effects on gout.

203 Among the four lipids we identified that only HDL was associated with gout (Fig. 2A-C and 204 Fig. 3). Specifically, the estimated odds ratio (OR) per standard deviation (SD) increase of 205 HDL $(\sim 12.26 \mathrm{mg} / \mathrm{dL})$ on gout was $0.75(95 \%$ CI $0.62 \sim 0.91, p=3.31 \mathrm{E}-3)$ and was 206 statistically significant after Bonferroni correction. The OR per SD increase of LDL ( 30.25 $207 \mathrm{mg} / \mathrm{dL})$, TC $(\sim 36.32 \mathrm{mg} / \mathrm{dL})$ or TG $(\sim 112.33 \mathrm{mg} / \mathrm{dL})$ for gout was $0.81(95 \% \mathrm{CI} 0.61 \sim 1.07$, $208 p=0.079), 0.98(95 \%$ CI $0.90 \sim 1.06, p=0.604)$ and $1.16(95 \%$ CI $0.93 \sim 1.45, p=0.186)$ 209 (Fig. 3 and Additional file 3: Table S5), respectively.

\section{Causal effect of lipids on serum urate}

211 We generated 87, 45, 57 and 49 lipid-specific instrumental variables for HDL, LDL, TC and 212 TG when evaluating their associations with serum urate. These instrumental variables 213 explicated a total of $5.0 \%, 3.0 \%, 2.5 \%$ and $3.7 \%$ phenotypic variances for HDL, LDL, TC 214 and TG, respectively (Additional file 2: Tables S6-S9). Thereafter, we used the random-effects 215 IVW method to estimate the causal effects due to the heterogeneity observed for all the four 216 sets of instruments (the $p$ values of the Cochran's Q test were 5.37E-10, 6.95E-04, 3.41E-06 217 and 2.67E-03 for HDL, LDL, TC and TG, respectively). HDL is negatively associated with 218 serum urate after Bonferroni correction (estimated causal effect $=-0.09,95 \%$ CI $-0.12 \sim-0.05$, $219 p=7.00 \mathrm{E}-04$ ) (Fig. 2D-F and Fig. 3). In addition, a positive association existed between TG 220 and serum urate (estimated causal effect $=0.10,95 \%$ CI $0.06 \sim 0.14, p=9.87 \mathrm{E}-05$ ) (Fig. 2G-I 
and Fig. 3). However, no association was observed between LDL/TC and serum urate

222 (Additional file 3: Table S5 and Fig. 3).

\section{Sensitivity analyses to validate the estimated causal effects}

224 We subsequently performed sensitivity analyses to validate the causal association observed 225 above. Herein, we relegated the results of serum urate to Additional file 4 and focused only on 226 gout in the following paragraphs. Due to the insignificant association between LDL/TC and 227 gout/serum urate, we only summarized their results in Fig. 3 and Additional file 3: Figures. 228 S1-S3, but did not pursue any of these two sets of traits further.

229 With the maximum likelihood method, the estimated OR per SD increase of HDL on gout was 2300.75 (95\% CI: $0.62 \sim 0.91, p=3.31 \mathrm{E}-03)$, in line with the IVW estimate; while the weighted 231 median method identified a consistently inverse but insignificant association (OR $=0.81,95 \%$

232 CI $0.59 \sim 1.11, p=0.197)$. The LOO analysis showed that none of the instruments of HDL 233 could individually affect the substantial causal effect estimates (Additional file 3: Figure S4).

234 However, Fig. 2A delineated that two instrumental variables of HDL with large effect sizes on 235 gout seemed to be potential outliers (i.e. rs2566091 in gene ALDH1A2 and rs9989419 in gene 236 AC012181.1). However, they did not exert any substantial impact on the estimated causal 237 effect of HDL on gout. Specifically, after removal, the OR was 0.74 (95\% CI $0.60 \sim 0.91, p=$ 238 4.18E-03), similar to that obtained with the instruments in total (Fig. 2B). Furthermore, the 239 funnel plot of HDL for gout exhibited a symmetric pattern around the causal effect point 240 estimates (Fig. 2C), offering little evidence for horizontal pleiotropy. The MR-Egger 241 regression also precluded the possibility of pleiotropy of instrument variables $($ the intercept $=$ $242-0.013,95 \%$ CI $-0.028 \sim 0.003, p=0.113)$. We further performed a multivariable Mendelian 243 randomization analysis to estimate the association between HDL and gout while adjusting for 244 LDL, TC and TG. The result showed HDL a de facto inverse relationship with gout even after 245 controlling for the residual three lipid traits (Additional file 3: Table S10), implying the 246 independent causal role of HDL in the risk of gout.

247 In the reverse causal analysis, we kept one instrumental variable for gout in estimation of its 248 causal effect on HDL, and 24 (or 22) instrumental variable of serum urate when estimating its 249 effect on HDL (or TG) (Additional file 2: Tables S11-S13). With the fixed-effects IVW 250 method, the causal effect of gout on HDL was estimated to be -0.019 (95\% CI: $-0.018 \sim$ 
251

252

253

254

255

256

257

258

259

260

261

262

263

264

265

266

267

268

269

270

271

272

273

274

275

276

277

278

3.20E-03, $p=0.173$ ), and the causal effects of serum urate on HDL and TG were estimated to be $-0.033(95 \%$ CI $-0.065 \sim-7.31 \mathrm{E}-04, p=0.045)$ and 0.016 (95\% CI: $-0.016 \sim 0.048, p=$ 0.330), respectively. All of estimates were insignificant in case of multiple hypothesis testing.

Therefore, this analysis excluded the probability of reverse causation from gout/serum urate to lipids, indicating that dyslipidemia (e.g. decreased HDL and/or increased TG) was a causal factor rather than a clinical manifestation of gout or serum urate.

\section{Results of the mediation analysis}

To estimate the possible mediation of serum urate in the progression from dyslipidemia and gout, we combined both the instrumental variables of lipids and serum urate, and reserved 116, 65, 76 and 73 lipid-specific instrumental variables for HDL, LDL, TC and TG in the mediation analysis (Additional file 2: Tables S14-S18). Notably, the following reported effects were corrected for the same scales (Additional file 1).

In the mediation analysis we observed that the summation of the mediation effect and the direct effect was approximately equal to the total effect (Table 2), indicating that our correction strategy used for those effects was justifiable. The total effect of HDL on gout was estimated to be -0.154 (95\% CI: $-0.256 \sim-0.051, p=3.31 \mathrm{E}-3$; Fig. 3) and the direct effect of HDL on gout was estimated to be -0.106 (95\% CI: $-0.189 \sim-0.022, p=1.35 \mathrm{E}-02)$. More importantly, the mediation effect of HDL on gout was estimated at -0.020 (95\% CI: -0.033 $-0.008, p=1.67 \mathrm{E}-03)$, which accounted for about $13.0 \%(=0.020 / 0.154)$ of the total effect, indicating that serum urate was a promising mediator between HDL and gout, and that HDL could indirectly affect the risk of gout via serum urate in addition to the direct impact. Likewise, the total effect of TG on gout was estimated at 0.082 (95\% CI: $-0.039 \sim 0.202, p=$ 1.86E-01) and the direct effect of TG on gout was estimated at 0.048 (95\% CI: $-0.057 \sim 0.152$, $p=3.71 \mathrm{E}-01)$. Furthermore, the mediation effect of TG on gout was estimated at $0.023(95 \%$ CI: $0.010 \sim 0.037, p=8.39 \mathrm{E}-04)$, accounting for about $28.0 \%(=0.023 / 0.082)$ of the total effect. TG also served as a mediator between TG and gout and could indirectly increase the risk of gout via serum urate. However, the mediation effects of serum urate between LDL/TC and gout were insignificant (Table 2). 


\section{Discussion}

\section{A summary of our study}

281 Many observational studies have reported that gout patients are often comorbid with 282 dyslipidemia $(15,69-72)$. However, the relationship between the two clinical disorders still 283 remains masked. To explore whether the association between dyslipidemia and gout is 284 causative, we performed a two-sample Mendelian randomization analysis in the present study. 285 To our best knowledge, this is the first study that attempts to investigate the causal 286 relationship between lipid/serum urate and gout by employment of summary association 287 statistics obtained from large-scale GWASs with Mendelian randomization within the 288 framework of instrumental variable approaches (20).

With the IVW method, we observed that HDL was negatively associated with gout and TG positively associated with serum urate. These inferred causal relationships were robust against

291 the designation of statistical methods. A series of sensitivity analyses (e.g., MR-Egger

292 regression) excluded the probability of instrumental pleiotropy that can contribute to bias in

293 the causal effect estimation. We also removed the reverse causality and confirmed that the 294 variations of HDL and TG levels were the premises of gout/serum urate rather than 295 consequences. Furthermore, the multivariable Mendelian randomization confirmed the 296 independent effect of HDL and TG on the gout/serum urate after adjustment for the other 297 lipids. With a mediation method, we identified a mediation effect between HDL and gout 298 mediated by serum urate, which indicated that elevated HDL could decrease the serum urate 299 and, in addition to the direct influence, indirectly relieved the risk of gout. Moreover, a 300 positive mediation effect was detected between TG and gout, i.e., though not a causal 301 contributor of gout, TG could indirectly exacerbate the risk of gout by elevating serum urate. 302 Additionally, we confirmed the positive association of serum urate with gout, which indicated 303 that elevated serum urate concentrations can causally increase the risk of gout. Overall, our 304 study affirmed that elevated HDL levels can directly and indirectly lead to the decreased risk 305 of gout, whereas elevation of TG levels can directly and indirectly elevate the risk of gout.

306 Mechanism underlying the association between dyslipidemia and gout/serum urate

307 The association between dyslipidemia and gout/serum urate is very complicated, implicated in 308 a number of mechanisms. Dyslipidemia may lead to an increase in ketone body and reduce 
the acid-discharging ability of the kidneys, with the renal arteries and the micro-arteries of the glomeruli impaired. Consequently, stenosis and occlusion of the diseased blood vessels

311 develop, with the excretion of uric acid from the kidneys is largely discounted (73).

312 Furthermore, hyperuricemia is reportedly associated with metabolic syndrome, which is

313 characteristic of hyperinsulinemia due to insulin resistance. An increase in TG or a decrease

314 in HDL may result in insulin resistance, consequently leading to the increased serum urate

315 during glycolysis and free fatty acid metabolism (74). Meanwhile, due to the increased

316 reabsorption of uric acid by the kidneys, serum urate directly contribute to hyperuricemia

317 (75).

318 The negative association between HDL and gout/serum urate may be attributable to the 319 anti-atherosclerotic, anti-oxidative, anti-inflammatory and anti-thrombotic effects of HDL 320 (76-78). Our mediation analysis revealed that, beyond the direct effect, HDL can directly 321 abate the risk of gout by decreasing serum urate concentration. The positive correlation 322 between TG and serum urate may be exemplified by the finding that TG promotes the 323 synthesis whereas decreases the normal excretion of serum urate. Indeed, TG can indirectly 324 affect the gout via serum urate. Since excessive intake of TG-rich food (e.g. fructose and 325 fat-rich food) may cause hyperthyroidism and further increase uric acid levels (79), overmuch 326 fat metabolism-related products will inhibit the excretion of serum urate. Furthermore, the 327 synthesis of triglycerides requires NADPH, which possibly increases the concentration of 328 serum urate (80). Intriguingly, clinical studies have validated that patients with hyperuricemia or gout have a $19 \%$ reduction in serum urate concentration at end of a three-week regimen of

330 oral lipid-lowering drugs, and serum urate concentration rebounded to its original level after 331 discontinuation of the agents (81), the mechanism for which awaits further investigation.

\section{$332 \quad$ Limitations}

333 Like other Mendelian randomization methods, our research has certain limitations. First, as

334 mentioned above, due to the small sample size of cases in gout GWAS, the statistical power 335 in our Mendelian randomized analysis was limited. For example, in our study, the sample size 336 of adult gout was 70,000 and the proportion of cases was only $3.1 \%$; the power calculation 337 results showed that we had a small to moderate power to detect the causal association 338 between lipids and gout (Fig. 4)(82). The estimated statistical power was $28 \%$ or $15 \%$ to 339 detect an OR of $0.70 / 1.30$ or $0.80 / 1.20$, respectively. Second, we hypothesized that the 
340 relationship between blood lipid levels and gout was linear in our analysis, but this linear

341 association might not be present in the clinical scenario, and thus we could not completely

342 eliminate the non-linear effects of lipids on gout. Third, as individual-level datasets were not

343 available; therefore, we could not evaluate the effects of extreme lipid levels on gout/serum

344 urate or conduct a stratified analysis (e.g. gender). In addition, again due to the unavailability

345 of individual-level data, we could not investigate the relationship between the TG-HDL ratio,

346 a known indicator of insulin resistance (83), and gout. Fourth, we adopted the multivariable

347 Mendelian randomization method to remove the effects of pleiotropic effects, but this method

348 was not applicable to the unknown or unmeasured pleiotropy (56).

349 Conclusion

350 In conclusion, our study confirmed the causal associations between HDL/TG and gout/serum

351 urate levels. Furthermore, serum urate can serve as a mediator for the effect of HDL or TG on 352 gout. 
354 Abbreviations

355 HDL: High-density lipoprotein cholesterol; GLGC: Global Lipids Genetics consortium;

356 GWASs: genome-wide association studies; IVW: inverse variance weighted; LDL:

357 Low-density lipoprotein cholesterol; LOO: leave-one-out; MR: Mendelian randomization; OR:

358 odds ratio; PVE: phenotypic variance explained; SD: standard deviation; SNPs: single

359 nucleotide polymorphisms; TC: Total cholesterol; TG: Triglyceride.

\section{Acknowledgements}

361 We are indebted to the GLGC and Global Urate Genetics Consortium studies for public 362 availability in making the summary data and we are grateful to all the investigators and 363 participants for their contributions to those studies. The data analyses in the present study 364 were supported by the high-performance computing at Xuzhou Medical University.

365 Authors' Contributions

366 PZ and SH conceived the design of the study; PZ and XY obtained the data; PZ and XY 367 cleared up the datasets; PZ and XY mainly performed the data analyses; HC, YG and JY 368 helped clear and analyze the data; PZ, XY and FG interpreted the results of the data analyses; $369 \mathrm{PZ}$ and XY drafted the manuscript, and all authors approved the manuscript and provided 370 relevant suggestions.

\section{Disclosure}

372 The authors declare that the research was conducted in the absence of any commercial or

373 financial relationships that could be construed as a potential conflict of interest.

\section{Funding}

375 This work was supported by Youth Foundation of Humanity and Social Science funded by 376 Ministry of Education of China (18YJC910002), the Natural Science Foundation of Jiangsu 377 (BK20181472), the General China Postdoctoral Science Foundation (2018M630607), the 378 Special China Postdoctoral Science Foundation (2019T120465), the Postdoctoral Science

379 Foundation of Xuzhou Medical University, QingLan Research Project of Jiangsu for 380 Outstanding Young Teachers and Six-Talent Peaks Project in Jiangsu Province of China 381 (WSN-087), the National Natural Science Foundation of China (81402765), the Statistical 382 Science Research Project from National Bureau of Statistics of China (2014LY112), the 383 Postgraduate Research \& Practice Innovation Program of Jiangsu Province (KYCX19_2250), 
medRxiv preprint doi: https://doi.org/10.1101/19006296; this version posted September 16, 2019. The copyright holder for this preprint (which

was not certified by peer review) is the author/funder, who has granted medRxiv a license to display the preprint in perpetuity.

All rights reserved. No reuse allowed without permission.

384 Social Development Project of Xuzhou, and the Priority Academic Program Development of

385 Jiangsu Higher Education Institutions (PAPD) for Xuzhou Medical University.

\section{Additional file}

387 Additional file 1: Supplementary Methods;

388 Additional file 2: Supplement Tables;

389 Additional file 3: Supplement Tables and Figures;

390 Additional file 4: Supplementary Results.

391 


\section{Reference}

1. Roddy E, Doherty M. Gout. Epidemiology of gout. Arthritis research \& therapy. 2010;12(6):223.

2. Roddy E, Choi HK. Epidemiology of gout. Rheum Dis Clin North Am. 2014;40(2):155-75.

3. Kuo C-F, Grainge MJ, Zhang W, Doherty M. Global epidemiology of gout: prevalence, incidence and risk factors. Nature Reviews Rheumatology. 2015;11(11):649-62.

4. Choi HK, Ford ES, Li C, Curhan G. Prevalence of the metabolic syndrome in patients with gout: the Third National Health and Nutrition Examination Survey. Arthritis Care \& Research: Official Journal of the American College of Rheumatology. 2007;57(1):109-15.

5. Choi HK, Atkinson K, Karlson EW, Curhan G. Obesity, weight change, hypertension, diuretic use, and risk of gout in men: the health professionals follow-up study. Archives of internal medicine. 2005;165(7):742-8.

6. Edwards NL. The role of hyperuricemia and gout in kidney and cardiovascular disease. Cleveland Clinic journal of medicine. 2008;75(5):S13.

7. De Vera MA, Rahman MM, Bhole V, Kopec JA, Choi HK. Independent impact of gout on the risk of acute myocardial infarction among elderly women: a population-based study. Annals of the rheumatic diseases. 2010;69(6):1162-4.

8. Krishnan E, Baker JF, Furst DE, Schumacher HR. Gout and the risk of acute myocardial infarction. Arthritis \& Rheumatism: Official Journal of the American College of Rheumatology. 2006;54(8):2688-96.

9. Lin K-C, Lin H, Chou P. The interaction between uric acid level and other risk factors on the development of gout among asymptomatic hyperuricemic men in a prospective study. The Journal of rheumatology. 2000;27(6):1501-5.

10. Duskin-Bitan H, Cohen E, Goldberg E, Shochat T, Levi A, Garty M, et al. The degree of asymptomatic hyperuricemia and the risk of gout. A retrospective analysis of a large cohort. Clinical rheumatology. 2014;33(4):549-53.

11. Zalokar J, Lellouch J, Claude JR, Kuntz D. Serum uric acid in 23,923 men and gout in a subsample of 4257 men in France. Journal of chronic diseases. 1972;25(5):305-12.

12. Choi HK, Curhan G. Gout: epidemiology and lifestyle choices. Current opinion in rheumatology. 2005;17(3):341-5.

13. Kuo CF, Grainge MJ, Zhang W, Doherty M. Global epidemiology of gout: prevalence, incidence and risk factors. Nature reviews Rheumatology. 2015;11(11):649-62.

14. Berkowitz D. Blood lipid and uric acid interrelationships. Jama. 1964;190(9):856-8.

15. Matsubara K, Matsuzawa Y, Jiao S, Takama T, Kubo M, Tarui S. Relationship between hypertriglyceridemia and uric acid production in primary gout. Metabolism: clinical and experimental. 1989;38(7):698-701.

16. Rho YH, Choi SJ, Lee YH, Ji JD, Choi KM, Baik SH, et al. The prevalence of metabolic syndrome in patients with gout: a multicenter study. Journal of Korean medical science. 2005;20(6):1029-33.

17. Rathmann W, Funkhouser E, Dyer AR, Roseman JM. Relations of hyperuricemia with the various components of the insulin resistance syndrome in young black and white adults: the CARDIA study. Annals of epidemiology. 1998;8(4):250-61.

18. Matsuo H, Yamamoto K, Nakaoka H, Nakayama A, Sakiyama M, Chiba T, et al. Genome-wide association study of clinically defined gout identifies multiple risk loci and its association with clinical subtypes. Ann Rheum Dis. 2016;75(4):652-9.

19. Tinahones F, Vazquez F, Soriguer F, Collantes E. Lipoproteins in patients with isolated hyperuricemia. Purine and Pyrimidine Metabolism in Man IX: Springer; 1998. p. 61-7. 
20. Davies NM, Holmes MV, Davey Smith G. Reading Mendelian randomisation studies: a guide, glossary, and checklist for clinicians. Br Med J. 2018;362.

21. Au Yeung SL, Luo S, Schooling CM. The Impact of Glycated Hemoglobin (HbA1c) on Cardiovascular Disease Risk: A Mendelian Randomization Study Using UK Biobank. Diabetes Care. 2018;41(9):1991.

22. Au Yeung SL, Lin SL, Lam HSHS, Schooling CM. Effect of 1-arginine, asymmetric dimethylarginine, and symmetric dimethylarginine on ischemic heart disease risk: A Mendelian randomization study. Am Heart J. 2016;182:54-61.

23. Davey Smith G, Ebrahim S. 'Mendelian randomization': can genetic epidemiology contribute to understanding environmental determinants of disease? Int $\mathrm{J}$ Epidemiol. 2003;32(1):1-22.

24. Hingorani A, Humphries S. Nature's randomised trials. Lancet. 2005;366(9501):1906-8. 25. Thanassoulis G, O'Donnell CJ. Mendelian randomization: nature's randomized trial in the post-genome era. JAMA. 2009;301(22):2386-8.

26. Mokry LE, Ross S, Ahmad OS, Forgetta V, Smith GD, Leong A, et al. Vitamin D and Risk of Multiple Sclerosis: A Mendelian Randomization Study. Plos Medicine. 2015;12(8). 27. Zeng P, Yu X, Xu H. Association between premorbid body mass index and amyotrophic lateral sclerosis: causal inference through genetic approaches. Frontiers in Neurology (in presss). 2019.

28. Zeng P, Zhou X. Causal effects of blood lipids on amyotrophic lateral sclerosis: a Mendelian randomization study. Human molecular genetics. 2019;28(4):688-97.

29. Holmes MV, Asselbergs FW, Palmer TM, Drenos F, Lanktree MB, Nelson CP, et al. Mendelian randomization of blood lipids for coronary heart disease. European heart journal. 2014;36(9):539-50.

30. Zeng P, Zhao Y, Qian C, Zhang L, Zhang R, Gou J, et al. Statistical analysis for genome-wide association study. Journal of Biomedical Research. 2015;29(4):285-97.

31. Zhao JV, Schooling CM. Effect of linoleic acid on ischemic heart disease and its risk factors: a Mendelian randomization study. BMC Med. 2019;17(1):61.

32. Disney-Hogg L, Cornish AJ, Sud A, Law PJ, Kinnersley B, Jacobs DI, et al. Impact of atopy on risk of glioma: a Mendelian randomisation study. BMC Med. 2018;16(1):42.

33. Bonilla C, Lewis SJ, Martin RM, Donovan JL, Hamdy FC, Neal DE, et al. Pubertal development and prostate cancer risk: Mendelian randomization study in a population-based cohort. BMC Med. 2016;14(1):66.

34. Larsson SC, Burgess S, Michaelsson K. Serum magnesium levels and risk of coronary artery disease: Mendelian randomisation study. BMC Med. 2018;16.

35. Willer CJ, Schmidt EM, Sengupta S, Peloso GM, Gustafsson S, Kanoni S, et al. Discovery and refinement of loci associated with lipid levels. Nat Genet. 2013;45(11):1274-83.

36. Köttgen A, Albrecht E, Teumer A, Vitart V, Krumsiek J, Hundertmark C, et al. Genome-wide association analyses identify 18 new loci associated with serum urate concentrations. Nature genetics. 2013;45(2):145.

37. Zeng P, Zhou X. Causal Association Between Birth Weight and Adult Diseases: Evidence From a Mendelian Randomization Analysis. Frontiers in Genetics. 2019;10(618).

38. Purcell S, Neale B, Todd-Brown K, Thomas L, Ferreira MAR, Bender D, et al. PLINK: A Tool Set for Whole-Genome Association and Population-Based Linkage Analyses. Am J Hum Genet. 2007;81(3):559-75.

39. Tyrrell J, Jones SE, Beaumont R, Astley CM, Lovell R, Yaghootkar H, et al. Height, body mass index, and socioeconomic status: mendelian randomisation study in UK Biobank. $\mathrm{Br}$ Med J. 2016;352:i582-i. 
40. Yeung CHC, Au Yeung SL, Fong SSM, Schooling CM. Lean mass, grip strength and risk 490 of type 2 diabetes: a bi-directional Mendelian randomisation study. Diabetologia. $491 \quad$ 2019;62(5):789-99.

492 41. Au Yeung SL, Schooling CM. Adiponectin and coronary artery disease risk: A bi-directional Mendelian randomization study. Int J Cardiol. 2018;268:222-6. 42. Larsson SC, Burgess S, Michaëlsson K. Association of Genetic Variants Related to Serum Calcium Levels With Coronary Artery Disease and Myocardial Infarction. JAMA. 2017;318(4):371-80. 43. Ahmad OS, Morris JA, Mujammami M, Forgetta V, Leong A, Li R, et al. A Mendelian randomization study of the effect of type-2 diabetes on coronary heart disease. Nat Commun. 2015;6:7060.

500 44. Robinson PC, Choi HK, Do R, Merriman TR. Insight into rheumatological cause and 501 effect through the use of Mendelian randomization. Nat Rev Rheumatol. 2016;12(8):486-96.

502 45. Censin JC, Nowak C, Cooper N, Bergsten P, Todd JA, Fall T. Childhood adiposity and 503 risk of type 1 diabetes: A Mendelian randomization study. PLoS Med. 2017;14(8):e1002362.

504 46. Østergaard SD, Mukherjee S, Sharp SJ, Proitsi P, Lotta LA, Day F, et al. Associations between Potentially Modifiable Risk Factors and Alzheimer Disease: A Mendelian Randomization Study. PLoS Med. 2015;12(6):e1001841.

508 Predisposition to Increased Blood Cholesterol and Triglyceride Lipid Levels and Risk of

509 Alzheimer Disease: A Mendelian Randomization Analysis. PLoS Med. 2014;11(9):e1001713.

510 48. Byrne EM, Ferreira MAR, Xue A, Lindstrom S, Jiang X, Yang J, et al. Is Schizophrenia a

511 Risk Factor for Breast Cancer?-Evidence From Genetic Data. Schizophr Bull. 2018.

512 49. Noyce AJ, Kia DA, Hemani G, Nicolas A, Price TR, De Pablo-Fernandez E, et al.

513 Estimating the causal influence of body mass index on risk of Parkinson disease: A Mendelian

514 randomisation study. Plos Medicine. 2017;14(6).

515 50. Zeng P, Yu X, Xu H. Association Between Premorbid Body Mass Index and Amyotrophic

516 Lateral Sclerosis: Causal Inference Through Genetic Approaches. Frontiers in Neurology.

$5172019 ; 10(543)$.

518 51. Burgess S, Small DS, Thompson SG. A review of instrumental variable estimators for Mendelian randomization. Statistical Methods in Medical Research. 2017;26(5):2333-55.

52. Bowden J, Smith GD, Haycock PC, Burgess S. Consistent Estimation in Mendelian Randomization with Some Invalid Instruments Using a Weighted Median Estimator. Genetic Epidemiology. 2016;40(4):304-14.

53. Burgess S, Butterworth A, Thompson SG. Mendelian Randomization Analysis With Multiple Genetic Variants Using Summarized Data. Genetic Epidemiology. 2013;37(7):658-65.

54. Burgess $S$, Thompson SG. Interpreting findings from Mendelian randomization using the MR-Egger method (vol 32, pg 377, 2017). Eur J Epidemiol. 2017;32(5):391-2.

55. Richmond RC, Davey Smith G. Commentary: Orienting causal relationships between two phenotypes using bidirectional Mendelian randomization. Int J Epidemiol. 2019.

56. Burgess S, Thompson SG. Multivariable Mendelian randomization: the use of pleiotropic genetic variants to estimate causal effects. American journal of epidemiology. 2015;181(4):251-60.

57. Drug and Therapeutics Bulletin Editorial Office. Latest guidance on the management of gout. BMJ. 2018;362:k2893.

58. MacKinnon DP, Fairchild AJ. Current directions in mediation analysis. Current directions in psychological science. 2009;18(1):16-20.

537 59. Imai K, Keele L, Tingley D. A general approach to causal mediation analysis. Psychol 
538 Methods. 2010;15(4):309.

539 60. MacKinnon DP. Introduction to statistical mediation analysis: Routledge; 2008.

540 61. MacKinnon DP, Fairchild AJ, Fritz MS. Mediation analysis. Annu Rev Psychol.

$541 \quad 2007 ; 58: 593-614$.

542 62. VanderWeele TJ. Mediation analysis: a practitioner's guide. Annu Rev Public Health. $543 \quad 2016 ; 37: 17-32$.

544 63. Zhan Y, Karlsson Ida K, Karlsson R, Tillander A, Reynolds Chandra A, Pedersen Nancy

545 L, et al. Exploring the Causal Pathway From Telomere Length to Coronary Heart Disease: A

546 Network Mendelian Randomization Study. Circ Res. 2017;121(3):214-9.

547 64. Burgess S, Daniel RM, Butterworth AS, Thompson SG. Network Mendelian

548 randomization: using genetic variants as instrumental variables to investigate mediation in 549 causal pathways. Int J Epidemiol. 2015;44:484-95.

550 65. Sobel ME. Asymptotic confidence intervals for indirect effects in structural equation 551 models. Sociological methodology. 1982;13:290-312.

552 66. Efron B, Tibshirani RJ. An introduction to the bootstrap: CRC press; 1994.

553 67. Greco M FD, Minelli C, Sheehan NA, Thompson JR. Detecting pleiotropy in Mendelian 554 randomisation studies with summary data and a continuous outcome. Statistics in medicine. 555 2015;34(21):2926-40.

556 68. Brockwell SE, Gordon IR. A comparison of statistical methods for meta-analysis. Stat 557 Med. 2001;20(6):825-40.

558 69. Emmerson B. Hyperlipidaemia in hyperuricaemia and gout. Annals of the rheumatic diseases. 1998;57(9):509-10.

70. Chen JH, Pan WH, Hsu CC, Yeh WT, Chuang SY, Chen PY, et al. Impact of obesity and hypertriglyceridemia on gout development with or without hyperuricemia: a prospective study. Arthritis care \& research. 2013;65(1):133-40.

563 71. Pascart T, Norberciak L, Ea HK, Guggenbuhl P, Liote F. GOSPEL 4 - Patients with early 564 onset gout develop earlier severe joint involvement and metabolic comorbid conditions. 565 Arthritis Care Res (Hoboken). 2018.

566 72. Peng T-C, Wang C-C, Kao T-W, Chan JY-H, Yang Y-H, Chang Y-W, et al. Relationship between hyperuricemia and lipid profiles in US adults. BioMed research international. $2015 ; 2015$.

73. Karalis DG, editor Intensive lowering of low-density lipoprotein cholesterol levels for primary prevention of coronary artery disease. Mayo Clinic Proceedings; 2009: Elsevier.

74. Lorenzo C, Okoloise M, Williams K, Stern MP, Haffner SM. The metabolic syndrome as predictor of type 2 diabetes: the San Antonio heart study. Diabetes care. 2003;26(11):3153-9. 75. Iwani NAKZ, Jalaludin MY, Zin RMWM, Fuziah MZ, Hong JYH, Abqariyah Y, et al. Triglyceride to HDL-C ratio is associated with insulin resistance in overweight and obese children. Scientific reports. 2017;7:40055.

576 76. Vitali C, Khetarpal SA, Rader DJ. HDL cholesterol metabolism and the risk of CHD: new insights from human genetics. Current cardiology reports. 2017;19(12):132.

578 77. Rosenson RS, Brewer Jr HB, Barter PJ, Björkegren JL, Chapman MJ, Gaudet D, et al. 579 HDL and atherosclerotic cardiovascular disease: genetic insights into complex biology. Nature Reviews Cardiology. 2018;15(1):9.

78. Loh TP, Poon M, Chan GHJ, Sethi SK, Wong A. Extremely low high-density lipoprotein cholesterol (HDL) in a patient with diffuse B-cell lymphoma. Pathology. 2017;49(5):550-1.

79. Giordano N, Santacroce C, Mattii G, Geraci S, Amendola A, Gennari C. Hyperuricemia and gout in thyroid endocrine disorders. Clin Exp Rheumatol. 2001;19(6):661-5.

586

80. Vuorinen-Markkola H, Yki-Järvinen H. Hyperuricemia and insulin resistance. The Journal of Clinical Endocrinology \& Metabolism. 1994;78(1):25-9. 
587 81. Feher M, Hepburn A, Hogarth M, Ball S, Kaye S. Fenofibrate enhances urate reduction in 588 men treated with allopurinol for hyperuricaemia and gout. Rheumatology. 2003;42(2):321-5.

589 82. Brion M-JA, Shakhbazov K, Visscher PM. Calculating statistical power in Mendelian 590 randomization studies. International journal of epidemiology. 2012;42(5):1497-501.

591 83. Keenan T, Blaha MJ, Nasir K, Silverman MG, Tota-Maharaj R, Carvalho JA, et al. 592 Relation of uric acid to serum levels of high-sensitivity C-reactive protein, triglycerides, and 593 high-density lipoprotein cholesterol and to hepatic steatosis. The American journal of 594 cardiology. 2012;110(12):1787-92.

595 84. Willer CJ, Schmidt EM, Sengupta S, Peloso GM, Gustafsson S, Kanoni S, et al. 596 Discovery and refinement of loci associated with lipid levels. Nat Genet. 597 2013;45(11):1274-83. 
medRxiv preprint doi: https://doi.org/10.1101/19006296; this version posted September 16, 2019. The copyright holder for this preprint (which

was not certified by peer review) is the author/funder, who has granted medRxiv a license to display the preprint in perpetuity.

All rights reserved. No reuse allowed without permission.

599 Table 1 Genetic data sets used in the present study

\begin{tabular}{cccc}
\hline Exposure(outcome) & Sample size(case/control) & Number of SNPs & Reference \\
\hline HDL & 99,900 & $2,260,809$ & $(84)$ \\
LDL & 95,454 & $2,251,395$ & $(84)$ \\
TC & 100,184 & $2,259,768$ & $(84)$ \\
TG & 96,598 & $2,252,990$ & $(84)$ \\
Gout & $69,374(2,115 / 67,259)$ & $2,538,056$ & $(36)$ \\
Serum urate & 110,347 & $2,450,547$ & $(36)$ \\
\hline
\end{tabular}

600 HDL, High-density lipoprotein cholesterol; LDL, Low-density lipoprotein cholesterol;

601 TC, Total cholesterol; TG, Triglycerides. 
Table 2 Mediation analysis of lipids on gout with serum urate concentrations

\begin{tabular}{|c|c|c|c|c|c|}
\hline & \multicolumn{3}{|c|}{ Mediation effect $(95 \% \mathrm{CI})$} & $\begin{array}{c}\text { Direct effect } \\
(95 \% \mathrm{CI})\end{array}$ & $\begin{array}{l}\text { Total effect } \\
(95 \% \text { CI })\end{array}$ \\
\hline LDL & $-0.010(-0.023 \sim 0.004)$ & $-0.010(-0.024 \sim 0.002)$ & 8.56 & $-0.090(-0.212 \sim 0.031)$ & $-0.117(-0.246 \sim 0.013)$ \\
\hline $\mathrm{TC}$ & $-0.007(-0.021 \sim 0.007)$ & $-0.007(-0.022 \sim 0.007)$ & 58.3 & $0.039(-0.007 \sim 0.084)$ & $-0.012(-0.058 \sim 0.033)$ \\
\hline
\end{tabular}

603 All the effects were corrected for the same scales.

604 HDL, High-density lipoprotein cholesterol; LDL, Low-density lipoprotein cholesterol; TC, Total cholesterol; TG, Triglyceride 